\title{
Atenção integral à saúde da população LGBT: Experiência de educação em saúde com agentes comunitários na atenção básica
}

\author{
Integral health care of LGBT population: Health education experience with community agents in \\ basic attention
}

\section{Atención integral la salud de la población LGBT: Experiencia de educación en salud con agentes comunitarios en la atención básica}

\author{
Mário Roberto Tavares Cardoso de Albuquerque ${ }^{1}\left({ }^{\circ}\right.$, Nara Macedo Botelho ${ }^{1} \mathbb{D}$, Cybelle Cristina Pereira Rodrigues ${ }^{2}$ \\ 1 Universidade do Estado do Pará (UEPA). Belém, PA, Brasil. \\ ${ }^{2}$ Centro Universitário do Estado do Pará (CESUPA). Belém, PA, Brasil.
}

\section{Resumo}

Objetivo: Relatar uma experiência de educação em saúde com agentes comunitários de saúde acerca da saúde da população LGBT na atenção básica. Métodos: Foi realizada uma ação de educação continuada por meio do estudo de casos escolhidos pelos agentes de saúde dentro de suas microáreas de atuação seguida de discussão em grupo e reflexão sobre as práticas vigentes. Resultados: Foram discutidos três casos apresentados pelos agentes de saúde que abordavam as temáticas de: (1) a escola e o preconceito; (2) a importância do apoio familiar e social; e (3) o papel da estratégia saúde da família e da educação em saúde. Percebeu-se que o tema permanece excluído das grandes discussões, sobretudo nas escolas médicas e na atenção básica, que é o primeiro contato do usuário com o Sistema Único de Saúde. Dessa forma, estratégias que objetivem discutir as peculiaridades da prevenção, promoção e assistência à saúde desses grupos devem ser estimuladas e reproduzidas tendo em vista uma melhor qualidade do atendimento a fim de captar esses pacientes em um ambiente favorável a práticas integrativas com respeito à diversidade sexual. Conclusão: Conclui-se que a atividade educacional realizada foi de extrema importância para desmistificar mitos e preconceitos envolvendo a saúde LGBT no cenário da atenção básica. Observou-se que após a atividade educacional os ACS se mostraram mais confiantes e sensibilizados sobre as temáticas abordadas e mudaram concepções no sentido de respeitar as decisões dos pacientes e realizar abordagem adequada para o acolhimento a esses usuários.

Palavras-chave: Direitos Sexuais e Reprodutivos; Minorias Sexuais e de Gênero; Homofobia; Educação Continuada; Agentes Comunitários de Saúde

Como citar: Albuquerque MRTC, Botelho NM, Rodrigues CCP. Atenção integral à saúde da população LGBT: Experiência de educação em saúde com agentes comunitários na atenção básica. Rev Bras Med Fam Comunidade. 2019;14(41):1758. https://doi.org/10.5712/rbmfc14(41)1758

\author{
Autor correspondente: \\ Mário Roberto Tavares Cardoso de \\ Albuquerque. \\ E-mail: mario-albuquerque@ hotmail.com \\ Fonte de financiamento: \\ declaram não haver. \\ Parecer CEP: \\ não se aplica. \\ Procedência e revisão por pares: \\ revisado por pares. \\ Recebido em: 26/05/2018. \\ Aprovado em: 09/03/2019.
}




\begin{abstract}
Objective: To report a health education experience with community health agents about the health of the LGBT population in basic care. Methods: A continuous education action was carried out by means of the study of cases chosen by health agents within their micro-areas of action followed by group discussion and reflection on current practices. Results: Three cases presented by the health agents that dealt with the themes of: (1) school and prejudice; (2) the importance of family and social support; and (3) the role of family health strategy and health education. It was noticed that the theme remains excluded from the great discussions, especially in medical schools and primary care, the first contact with the Brazilian health system (SUS), thus strategies that aim to discuss the peculiarities of prevention, promotion and health care of these groups should be stimulated and reproduced with a view to a better quality of care in order to capture these patients in an environment favorable to integrative practices with respect to sexual diversity. Conclusion: It was concluded that the educational activity performed was extremely important to demystify myths and prejudices involving LGBT health in the primary care setting. It was observed that after the educational activity the CHAs were more confident and sensitized on the topics addressed and changed conceptions in order to respect the decisions of the patients and to carry out an appropriate approach to the reception of these users.
\end{abstract}

Keywords: Reproductive Rights; Sexual and Gender Minorities; Homophobia; Education, Continuing; Community Health Workers

\title{
Resumen
}

Objetivo: Informar una experiencia de educación en salud con agentes comunitarios de salud acerca de la salud de la población LGBT en la atención básica. Métodos: Se realizó una acción de educación continuada por medio del estudio de casos escogidos por los agentes de salud dentro de sus microáreas de actuación seguido de discusión en grupo y reflexión sobre las prácticas vigentes. Resultados: Hemos discutido tres casos presentados por los funcionarios de salud que abordaron los siguientes temas: (1) la escuela y el prejuicio; (2) la importancia del apoyo familiar y social; y (3) el papel de la estrategia sanitaria de la familia y de la educación en salud. Se percibió que el tema permanece excluido de las grandes discusiones, sobre todo en las escuelas médicas. Estrategias que objetiven discutir las peculiaridades de la promoción y asistencia a la salud de esos grupos deben ser estimuladas teniendo en vista una mejor calidad de la atención a fin de captar esos pacientes en un ambiente favorable a prácticas integrativas con respecto a la diversidad sexual. Conclusión: Se concluye que la actividad educativa realizada fue de extrema importancia para desmitificar mitos y prejuicios involucrando la salud LGBT en el escenario de la atención básica. Se observó que después de la actividad educativa los ACS se mostraron más confiados y sensibilizados sobre las temáticas abordadas y cambiaron concepciones en el sentido de respetar las decisiones de los pacientes y realizar un abordaje adecuado para la acogida a esos usuarios.

Palabras clave: Derechos Sexuales y Reproductivos; Minorías Sexuales y de Género; Homofobia; Educación Continua; Agentes Comunitarios de Salud

\section{Introdução}

Um estudo de meta-análise realizada a partir de 25 estudos internacionais identificou que homens gays e bissexuais estão quatro vezes mais propensos a referir tentativas de suicídio comparados aos heterossexuais, e mulheres lésbicas e bissexuais possuem duas vezes mais chances de tentar o suicídio em relação às heterossexuais. ${ }^{1}$ Por sua vez, estimativas dessa tentativa são superiores a $30 \%$ em indivíduos transgêneros, muito superior aos $8,7 \%$ encontrados na população cisgênero. ${ }^{2}$

Remy et al. ${ }^{3}$ demonstraram que entre os 240 sujeitos incluídos em sua pesquisa a prevalência de sintomas de depressão nos últimos 12 meses no grupo não heterossexual foi $37 \%$ superior à dos heterossexuais, sugerindo que estratégias devem ser desenvolvidas para avaliar e abordar as necessidades individuais, e as abordagens de tratamento devem ser adaptadas para sintomas depressivos em jovens, não heterossexuais, dependentes químicos.

Outro agravante diz respeito ao fato de que práticas homoafetivas permanecem ilegais em 78 países e, em cinco deles, são punidas com sentença de morte. No Brasil, segundo a Secretaria de Direitos Humanos da Presidência da República, em 2012 registravam-se em torno de 27 queixas por dia relacionadas à discriminação sexual, ressaltando que os dados poderiam ser ainda mais alarmantes devido à subnotificação. ${ }^{4,5}$ 
Destaca-se que, até o século XIX, as pessoas de mesmo sexo biológico se relacionavam sexual e eroticamente, mas não eram chamadas homossexuais e, portanto, não se sentiam enquanto tais. Assim, as práticas homoeróticas existiam, mas não existiam a homossexualidade e a heterossexualidade como formas distintas de vivência da sexualidade humana. ${ }^{6}$

A necessidade de uma política de saúde para a população LGBT já fora evidenciada e formalizada no Brasil em 2004, quando da elaboração e apresentação do "Brasil sem Homofobia (BSH)" - Programa de Combate à Violência e à Discriminação contra LGBT e de Promoção da Cidadania. Esse programa do Governo Federal se situava no âmago das políticas públicas para a população em questão, visando o combate ao preconceito e às intolerâncias, que têm como consequência iniquidades e falta de garantia de direitos fundamentais a gays, lésbicas, bissexuais, travestis e transexuais. ${ }^{7,8}$

No início, o Brasil Sem Homofobia (BSH) esteve mais voltado para questões de violência física e assassinatos de homossexuais. Porém, logo investiu em outros temas, como, por exemplo, a cidadania, problematizando a questão da desigualdade de direitos, identificada já no próprio título do $\mathrm{BSH}$, o que representou um avanço no tratamento da questão por parte do governo e da agenda LGBT com a perspectiva de justiça social. ${ }^{9}$

É valido ressaltar que essa política destaca em seus capítulos $\mathrm{V}$ e VI o direito à educação e o direito à saúde, consolidando atenção e tratamentos igualitários. O direito à saúde, por sua vez, teve como desdobramento o lançamento, em 2010, pelo Ministério da Saúde, da Política Nacional de Atenção Integral à População LGBT, que tem como fundamento a implementação de ações para eliminar a discriminação contra lésbicas, gays, bissexuais, transexuais e travestis, e afirma que este deve ser um compromisso ético-político para todas as instâncias do SUS, de seus gestores, conselheiros, de técnicos e de trabalhadores de saúde. ${ }^{10}$

A despeito do que preconiza essa política, ainda pouco se observa de interesse e incentivo das diversas esferas de gestão e dos profissionais de saúde em trazer o tema para discussão nos variados setores. Nesse contexto a atenção básica, por ser o primeiro contato do cidadão com o sistema de saúde, e tendo como pilares o acolhimento integral e a continuidade do cuidado, projeta-se como ambiente propício para ações de educação em saúde.

Ressalta-se nesse cenário o papel do Agente Comunitário de Saúde (ACS), que por ser elemento fundamental, o elo que une diretamente a comunidade com a equipe de saúde, deve estar bem esclarecido e orientado a abordar e acolher pacientes LGBT. Prática esta ainda pouco desenvolvida e priorizada na atenção básica, na qual esses profissionais recebem pouca qualificação comparado a outras temáticas como os programas de hipertensão e diabetes, pré-natal ou imunização.

Dessa forma, o presente estudo visa contribuir com a literatura científica ao relatar uma experiência exitosa e factível de reprodutibilidade envolvendo a educação em saúde dos ACS, sensibilizando-os e pondo em evidência a necessidade de incremento da temática no planejamento e rotina da estratégia saúde da família. 


\section{Objetivo}

Este relato de experiência teve por objetivo descrever as reflexões obtidas em uma atividade de educação continuada sobre saúde da população LGBT realizada no âmbito da atenção básica com agentes comunitários de saúde (ACS).

\section{Métodos}

Trata-se de um relato de experiência vivenciado pelo autor da pesquisa durante uma atividade de educação em saúde previamente agendada dentro da rotina de reuniões de equipe de uma Estratégia Saúde da Família (ESF).

Foi ministrada uma capacitação com agentes comunitários de saúde (ACS) de uma ESF em Ananindeua/PA no ano de 2018, baseada em casos reais apresentados pelos ACS, cujo tema central era a saúde integral da população LGBT.

O fator motivador para a execução da atividade veio dos próprios agentes de saúde, que, em diversas situações, manifestavam o seu desejo em colaborar de forma mais eficiente com pacientes LGBT, porém se sentiam inseguros para abordá-los e com lacunas de conhecimento por falta de qualificações sobre o tema.

Essa atividade tinha como objetivos: identificar casos de pessoas vulneráveis na área de abrangência da ESF, compreender os principais conceitos referentes à temática desenvolvida, sensibilizar os ACS sobre as dificuldades e necessidade de cuidado da população LGBT e capacitá-los para promover o acolhimento adequado e captação para consulta dessa população.

As reflexões apresentadas foram concebidas por meio de grupo focal, que em geral permite melhor contextualização dos fatos, por meio da criação de uma situação interativa entre os participantes, mais próxima do que uma entrevista individual.

A atividade contou com cinco ACS, duas do sexo feminino e três do sexo masculino, membros de uma ESF de Ananindeua/PA em que o autor principal era o médico, em um universo de seis ACS. Um deles não participou por se encontrar em período de férias durante a prática.

No dia da reunião, em um primeiro momento foi feito uma familiarização com termos referentes à saúde LGBT a exemplo do significado das palavras gay, lésbica, bissexual, transgênero, cisgênero, homossexual, travesti, entre outros, além de solicitar autorização dos mesmos para gravar os relatos da capacitação. Posteriormente, cada ACS apresentou um caso de sua área e, por fim, fez-se uma discussão em grupo com reflexões acerca do atendimento integral à população em questão.

Este texto, ainda que se trate de relato de experiência, está em consonância com as normas éticas vigentes para publicação de artigos. Foi obtido posteriormente o consentimento livre e esclarecido por escrito dos participantes da atividade, todos maiores de 18 anos, autorizando a gravação e divulgação do relato da atividade, com garantia de sigilo e confidencialidade da identidade dos participantes. 


\section{Resultados e Discussão}

\section{Caso 1 - A escola e o preconceito}

Embora tenha sido solicitado aos cinco agentes comunitários de saúde que cada um trouxesse um caso de sua área para discussão, apenas três trouxeram seus relatos para a atividade, as duas agentes do sexo feminino e um agente do sexo masculino. Os demais, embora tenham comparecido, alegaram que não tinham casos que se enquadrassem na temática LGBT. Tal fato pode ser explicado ou pela falta de conhecimento do ACS sobre a sua população adstrita ou pela dificuldade de abertura e comunicação dos pacientes sobre questões referentes à sexualidade, principalmente com homens, o que é salientado por diversos estudos na literatura científica. ${ }^{1,3,7-9}$

Além disso, é válido destacar o duplo papel do ACS, que é, ao mesmo tempo, profissional assistente de saúde e membro da comunidade; dessa forma, ocorre interferência de relações afetivas que podem dificultar a abordagem ou ainda inibir o acesso a este tema na prática diária.

O primeiro caso relatado foi de um adolescente do sexo masculino, 15 anos, pardo, com condição financeira restrita, que frequentava uma escola pública de ensino fundamental do município. A problemática apresentada era a de que o mesmo sofria preconceito na escola por se declarar abertamente homossexual e apresentar comportamentos tidos como "femininos". Isso chegou ao conhecimento de sua genitora e trazia muitas preocupações relacionadas a possíveis agressões físicas e verbais na escola e na vizinhança.

Tal angústia se justifica pela população LGBT estar mais suscetível a agressões comparado a população heterossexual. Estudo realizado pela Associação da Parada do Orgulho GLBT de São Paulo, em 2006, com uma amostra de 846 participantes, evidenciou que $67 \%$ dos respondentes declararam haver sofrido discriminação devido à sua sexualidade, bem como $59 \%$ dos sujeitos já haviam sido vítimas de agressão pelo mesmo fator determinante. ${ }^{8}$

Esta situação está cada dia menos tolerável, pois cresce o número de jovens LGBT que vivem abertamente sua sexualidade, o que leva ao maior conhecimento da falta de cidadania sexual e, portanto, da persistência de condições de desigualdade e injustiça social. Além disso, a abertura social que leva esses adolescentes a não quererem "esconder-se" por mais tempo não se acompanha de mudanças no sistema educativo, no que se pode deduzir que os adolescentes ficam mais expostos à homofobia dentro das escolas. ${ }^{11}$

Durante a discussão, os ACS se mostraram solidários ao caso, criticando o fato de ainda haver preconceito de qualquer espécie no mundo e ainda mais em um país multicultural e diversificado como o Brasil. Refletiram sobre o papel da escola neste sentido, pois possuem filhos matriculados nas mesmas escolas dos pacientes que assistem, e não houve relatos de atividades de combate ao bullying, ao preconceito, no ambiente escolar. 
O processo de construção de práticas, relações e representações de gênero-sexualidade ocorre em diferentes espaços sociais: na família, na comunidade, no trabalho e, também, na escola. Mesmo que não figure em nenhuma diretriz educacional que a escola deve ensinar meninos a serem meninos e meninas a serem meninas, esse parece ser um conteúdo curricular assumido, trabalhado e priorizado por estas instituições. Há muito mais sendo ensinado do que o que está escrito nos projetos político-pedagógicos e planejamentos de aula. A divisão por sexo nas aulas de Educação Física; o quadro de chamada e as filas de meninos e de meninas na educação infantil são alguns exemplos da expressão da heteronormatividade. ${ }^{12}$

No caso reportado tem-se um adolescente que vive abertamente sua sexualidade. Porém, essa ainda não é a regra. Outra forma de sofrimento decorrente da discriminação é o medo de rejeição e a consequente internalização da sexualidade, que tem sido denominado de "homofobia internalizada". ${ }^{13}$

Estudos apontam que, embora a maioria dos jovens LGBT sejam saudáveis físico e psicologicamente, ainda existe um grupo expressivo que está mais sujeito a infecções sexualmente transmissíveis, a exemplo do HIV, obesidade, abuso de substâncias licitas e ilícitas, transtornos de humor e ansiedade, entre outras afecções. Além disso, estes jovens apresentam medo de procurar serviços de saúde e são mais suscetíveis a viverem sozinhos em consequência de abandono familiar. ${ }^{14}$

As reflexões geradas na capacitação chegaram à conclusão de que intervenções sistemáticas deveriam ser realizadas, envolvendo sujeitos políticos, a escola, as unidades de saúde e a família, visando o estabelecimento de estratégias que promovam um ambiente escolar e comunitário saudável para o desenvolvimento desses adolescentes.

Os agentes de saúde citaram, por exemplo, a necessidade de realizar consultas familiares para entender a visão do paciente nesse contexto, integrar palestras e atividades dentro do Programa Saúde na Escola (PSE), além de realizar qualificações com os ACS de outras equipes e se possível promovida pela Secretaria de Saúde com todas as ESF.

\section{Caso 2 - A importância do apoio familiar e social}

O segundo caso relatado foi de um rapaz de 19 anos, negro, de condição financeira restrita. AACS relatou que o mesmo tinha sido agredido e expulso de casa pelos pais ainda na adolescência por não aceitarem a sexualidade do filho. Desde então, o rapaz passou a morar em um apartamento com outros dois colegas, realizou tentativa de suicídio, abandonou os estudos e sua remuneração vinha de "programas sexuais" realizados como travesti. AACS relatou ainda que o mesmo tinha sido diagnosticado com infecção pelo HIV em torno de um ano antes e fazia acompanhamento em um centro de referência do município.

Soliva e Silva Junior ${ }^{15}$ destacam que as relações domésticas são reveladoras de pesadas estruturas de poder que configuram um esforço de heterossexualização compulsória. Esse esforço faz com que comportamentos considerados não conformados às expectativas familiares acerca da sexualidade e do gênero sejam controlados pelos familiares visando à reintegração desses jovens à norma sexual dominante ora sob o pretexto de garantir uma melhor qualidade de vida para os jovens, ora preocupando-se com a repercussão no ambiente familiar e social.

Os autores ressaltam ainda que várias são as estratégias empregadas pelos pais para contornarem esse suposto "problema". As violências físicas e psicológicas aparecem como modalidades constantemente adotadas para este fim. 
Essas observações são evidenciadas no relato apresentado, em que a dificuldade e recusa de aceitação familiar levou o paciente a situações de violência e abandono, o que provavelmente contribuiu para o abandono escolar e inserção no mercado sexual.

É válido destacar a percepção e sensibilização da ACS que relatou este caso. Por vezes, ela se emocionou bastante e demonstrou indignação com a expulsão do paciente do núcleo familiar e as repercussões que esse fato trouxeram a sua vida.

Em diversos momentos ela foi apoiada pelos demais colegas que conjuntamente abordaram e destacaram a importância de um núcleo familiar bem estruturado e do combate ao preconceito como estratégias fundamentais para garantir a saúde de pacientes LGBT.

A literatura ${ }^{7}$ demonstra que devido à rejeição e à discriminação existente no meio social, nas escolas, nas famílias, enfim, nas instituições sociais, torna-se difícil para o adolescente assumir-se como não heterossexual, e quando o faz perante a família, dificilmente encontra acolhimento e respeito. A família, portanto, que poderia se constituir como promotora do bem-estar, antagonicamente colaborou para o desencadeamento de situações constrangedoras e dolorosas para esse jovem, que buscou apoio em suas redes de amizades.

Assim, o espaço familiar, que deveria ser acolhedor, inserindo o jovem na dinâmica da sociedade e ofertando segurança, torna-se um ambiente hostil e que colabora para o sofrimento físico e psíquico da população LGBT.

Levantou-se ainda, durante a discussão, a questão da tentativa de suicídio, que é a segunda principal causa de morte violenta em jovens no mundo, ${ }^{16}$ tornando o tópico do suicídio em adolescentes um problema de saúde pública e que merece prioridade pelas equipes de assistência à saúde.

É importante salutar que nesta abordagem foi o momento em que mais se percebeu mudança de conduta dos agentes que se encontravam mais relutantes, mais tradicionalistas. Pois, apesar de seus princípios religiosos os fazerem enxergar a homossexualidade como inadequada, não aceitavam que este fato pudesse levar alguém a infelicidade, a pensar em ceifar a própria vida. Pois a vida seria um dom e todos teriam direito a serem felizes e não sofrerem julgamentos pela orientação sexual.

Adolescentes lésbicas, gays, bissexuais e transgêneros estão mais propensos a contemplar, planejar e tentar de fato o suicídio comparado a seus pares heterossexuais, fato que já está documentado em nível global. ${ }^{1-3,16}$

Um estudo brasileiro 4 apontou que os efeitos do estigma e da discriminação constituem importantes barreiras para que homossexuais procurem serviços de saúde e realizem testes diagnósticos para HIV. Felizmente, no caso exposto, embora o paciente não aceitasse o atendimento da unidade de saúde, ele se dispôs a realizar o teste em algum momento de sua trajetória e fazia acompanhamento e tratamento em centro especializado.

No caso dos pacientes HIVIAIDS, a discriminação resulta em uma barreira ainda maior para a prevenção e assistência à saúde, retardando diagnóstico e tratamento e levando muitas vezes à não aderência terapêutica. Estudos sugerem que treinamentos específicos, como por exemplo a educação em saúde na atenção básica realizada neste relato, podem resultar em melhores práticas da equipe de saúde em assistir esses pacientes, reduzindo estigmas e discriminação. ${ }^{17}$ 
Trabalhos demonstram que nas últimas décadas a aceitação sexual nas sociedades ocidentais tem aumentado, o que reflete em ganhos para as minorias como leis para punir práticas discriminatórias e legalidade do casamento entre pessoas do mesmo sexo em mais de 20 países. ${ }^{18} \mathrm{Na}$ atividade educativa foi possível observar boa tolerância dos ACS, que se mostraram sensibilizados com o caso reportado e compreenderam a importância do apoio familiar e social para evitar desfechos como o do paciente em questão.

\section{Caso 3 - O papel da estratégia saúde da família e da educação em saúde}

O terceiro e último caso discutido dizia respeito a uma paciente do sexo feminino de 34 anos, parda, sem filhos, com emprego fixo, abertamente homossexual, e que morava com uma companheira. O principal tópico que o agente gostaria de discutir era o fato da paciente se negar a aceitar consultas e acompanhamento na unidade.

Ele relatou que já tinha feito diversos convites e tentativas de orientação, mas que a paciente não aceitava, até que em determinada ocasião ela referiu que deixou de ir a unidade de saúde a partir de uma experiência em que tinha conversado com uma funcionária do posto de saúde para agendar exame preventivo do câncer do colo do útero (PCCU) e foi informada que no seu caso o exame não era necessário por ser homossexual. Relatou que tal fato a agrediu, pois sabia que a orientação não estava correta e se sentiu discriminada e desde então sempre fez consultas pelo plano de saúde.

O Sistema Único de Saúde, instituído pela Lei Federal № 8.080/90, tem como princípios a universalidade do acesso e a integralidade da atenção, em que grupos e setores mais vulneráveis devem ser priorizados.

Nesse contexto, comparado com a população heterossexual, as pessoas LGBT estão mais sujeitas a problemas físicos, psíquicos e emocionais, a exemplo de transtornos de humor e ansiedade, abuso de álcool e drogas e desordens de autoimagem. Dessa forma, é possível inferir que essa população possuir maior demanda de assistência à saúde e, portanto, os profissionais devem estar aptos e qualificados a recebê-los. ${ }^{2,19}$

Indivíduos LGBT são mais relutantes a procurar o serviço de saúde, como observado neste relato, em decorrência do medo de discriminação. Tal receio se mostra extremamente cabível, pois práticas discriminatórias têm sido demonstradas em todos os níveis, desde estudantes até profissionais formados na área da saúde. ${ }^{20}$ Poucos são os trabalhos em que os pacientes referem que sua condição sexual foi vista com neutralidade ou como motivadora para um melhor atendimento e cuidado. ${ }^{21}$

Esses resultados apontam a necessidade de desenvolver políticas públicas e programas voltados as minorias sexuais que abordem as nuances da assistência em saúde e considerem os reflexos da identidade gênero na qualidade de vida dessa população. ${ }^{22}$

AESF está na linha de frente do cuidado a essa população, por ser a porta de entrada do sistema de saúde e estar sediada em tese dentro da comunidade e próximo ao domicílio de sua população adscrita. Porém, o que se encontra na prática são equipes sem preparo adequado e que não fornecem um ambiente acolhedor que respeite e perceba a população LGBT enquanto grupo vulnerável, a exemplo da unidade de saúde em que ocorreu este relato. ${ }^{10}$ 
Embora a unidade tenha sido inaugurada há quase dez anos e sediasse três estratégias de saúde da família, a capacitação era a primeira iniciativa já feita priorizando a população LGBT. Fato que não passou despercebido pelos ACS, que inclusive ressaltaram que já tinham feito inúmeras ações de saúde com pacientes hipertensos, diabéticos, em saúde da mulher, por vezes repetitivas e com a mesma população, e que poderiam dar lugar em algum momento para discussões sobre a temática abordada.

A predileção por outros temas provavelmente vem de escolha dos próprios profissionais médicos e de enfermagem que atuam na atenção básica, pois geralmente são estes que organizam e definem a temática a ser abordada e, via de regra, são melhor capacitados e se sentem mais confortáveis em tratar de assuntos que são priorizados desde a faculdade, o que deixa a temática de saúde da população LGBT marginalizada.

Um estudo que corrobora essa percepção foi realizado em 2011 em 132 escolas médicas do Canadá e Estados Unidos e demonstrou que durante os quatro anos de academia em medicina, somente cinco horas eram dedicadas a conteúdos voltados à saúde da população LGBT. ${ }^{23}$

Uma recomendação recorrente nos artigos encontrados é a integração da saúde da população LGBT aos currículos das escolas médicas e de enfermagem tanto em nível de graduação quanto de pós-graduação. Essa integração proporcionaria maior carga horária para estudo das peculiaridades que envolvem a assistência em saúde nessa área, assim como a visão dos alunos de forma mais natural e conjunta dentro dos cenários de prática, em contraponto à atual concepção de que a saúde LGBT seria um tópico com abordagem restrita e unifocal. ${ }^{17}$

Essa visão integral também foi partilhada pelos agentes de saúde durante a capacitação. As reflexões obtidas ao final da atividade apontaram para a necessidade de uma assistência a saúde integrada entre os diversos profissionais da ESF e que também respeite e considere os desejos do paciente LGBT e suas peculiaridades. Essa abordagem é conhecida como tomada de decisão compartilhada e tem sido positivamente associada com melhor satisfação e adesão do paciente aos tratamentos propostos e maior qualidade do cuidado. ${ }^{24}$

\section{Conclusão}

Embora iniciativas mundiais e nacionais como a Política Nacional de Atenção Integral a Saúde da População LGBT do Ministério da Saúde preconizem a qualificação e discussão dessa temática no âmbito da saúde e educação, o tema permanece marginalizado e excluído das grandes discussões, sobretudo nas escolas médicas e na atenção básica, primeiro contato do usuário do SUS com o sistema único de saúde.

Estratégias que objetivem discutir as peculiaridades da prevenção, promoção e assistência à saúde desses grupos devem ser estimuladas e reproduzidas, tendo em vista uma melhor qualidade do atendimento, a fim de captar esses pacientes em um ambiente favorável a práticas integrativas com respeito a diversidade sexual.

As reflexões oriundas deste relato de experiência podem contribuir para a formação ética e capacitação dos agentes comunitários de saúde (ACS) e, dessa forma, melhorar o atendimento ao paciente LGBT. Não se pode esquecer ainda que o ACS é peça fundamental na atenção básica por possuir proximidade com a comunidade e ser importante disseminador de conhecimento, permitindo transferir e aplicar o aprendizado das capacitações em suas áreas de atuação. 
Um elemento importante a ser destacado é o entendimento que ACS, embora seja um profissional da equipe de saúde, também é membro da comunidade, e, portanto, durante a atividade percebeu-se a existência de vínculos afetivos difíceis de serem dissociados. Prevaleceram influências geradoras de benefícios como empatia e solidariedade, porém por vezes também se pode observar críticas, sobretudo com embasamento da religiosidade e suas doutrinas, ainda enxergando a prática sexual entre pessoas do mesmo gênero como inadequada e que não deveria ser estimulada.

Planejar e reformular a política de cuidados a grupos vulneráveis como as minorias sexuais deve ser prioridade nas instituições formativas e assistenciais em saúde. Algumas mudanças são sutis e facilmente aplicáveis como a mudança do vocabulário e o olhar integral do paciente. Outras necessitam reflexões mais profundas e transformações culturais, que implicam em modificações nas práticas educacionais vigentes.

Conclui-se que a atividade educacional realizada foi de extrema importância para desmistificar mitos e preconceitos envolvendo a saúde LGBT no cenário da atenção básica. Os ACS durante puderam entender as diferenças entre termos geralmente considerados básicos como diferenciar homossexualidade de homossexualismo, transexual, cisgênero e transgênero, entre outros.

Observou-se que após a atividade educacional os ACS relataram estar mais confiantes e sensibilizados sobre as temáticas abordadas e mudaram concepções no sentido de respeitar as decisões dos pacientes e realizar abordagem adequada para acolhimento a esses usuários, mesmo que as vezes possuíssem ideologias próprias contrárias a esta prática sexual, mas que de fato não deveriam interferir na assistência à saúde, reconhecendo os riscos da falta de assistência como relatado nos casos analisados.

\section{Conflito de interesses}

Declaram não haver.

\section{Contribuição dos autores}

Concepção e/ou delineamento do estudo: MRTCA. Aquisição, análise e interpretação dos dados: MRTCA. Revisão crítica da versão preliminar: NMB, CCPR.

Todos os autores aprovaram a versão final e concordaram com prestar contas sobre todos os aspectos do trabalho.

\section{Agradecimentos}

Agradecimento a toda equipe do mestrado profissional em ensino em saúde na Amazônia da Universidade do Estado do Pará.

\section{Referências}

1. Haas AP, Lane A; Working Group for Postmortem Identification of SO/GI. Collecting Sexual Orientation and Gender Identity Data in Suicide and Other Violent Deaths: A Step Towards Identifying and Addressing LGBT Mortality Disparities. LGBT Health. 2015;2(1):84-7. https://doi.org/10.1089/lgbt.2014.0083

2. Rood BA, Puckett JA, Pantalone DW, Bradford JB. Predictors of Suicidal Ideation in a Statewide Sample of Transgender Individuals. LGBT Health. 2015;2(3):270-5. https://doi.org/10.1089/lgbt.2013.0048

3. Remy LS, Scherer J, Guimarães L, Surratt HL, Kurtz SP, Pechansky F, et al. Anxiety and depression symptoms in Brazilian sexual minority ecstasy and LSD users. Trends Psychiatry Psychother. 2017;39(4):239-46. https://doi.org/10.1590/2237-6089-2016-0081 
4. Magno L, Dourado I, Silva LAV, Brignol S, Brito AM, Guimarães MDC, et al. Factors associated with self-reported discrimination against men who have sex with men in Brazil. Rev Saúde Pública. 2017;51:102. https://doi.org/10.11606/S1518-8787.2017051000016

5. Byne W. LGBT Health Equity: Steps Toward Progress and Challenges Ahead. LGBT Health. 2015;2(3):193-5. https://doi.org/10.1089/ Igbt.2015.0084

6. Dieter CT. As raízes históricas da homossexualidade, os avanços no campo jurídico e o prisma constitucional. Rio de Janeiro; 2011. [acesso 2019 Jan 6]. Disponível em: http://www.ibdfam.org.br/_img/artigos/As\%20raízes\%20históricas\%2012_04_2012.pdf

7. Perucchi J, Brandão BC, Vieira HIS. Aspectos psicossociais da homofobia intrafamiliar e saúde de jovens lésbicas e gays. Estud Psicol (Natal). 2014;19(1):67-76. https://doi.org/10.1590/S1413-294X2014000100009

8. Lionço T. Que direito à saúde para a população GLBT? Considerando direitos humanos, sexuais e reprodutivos em busca da integralidade e da eqüidade. Saude Soc. 2008;17(2):11-21. https://doi.org/10.1590/S0104-12902008000200003

9. Vianna CP. O movimento LGBT e as políticas de educação de gênero e diversidade sexual: perdas, ganhos e desafios. Educ Pesqui. 2015;41(3):791-806. https://doi.org/10.1590/s1517-97022015031914

10. Motta JIJ. Sexualidades e políticas públicas: uma abordagem queer para tempos de crise democrática. Saúde Debate. 2016;40(no. spe):73-86. https://doi.org/10.1590/0103-11042016s07

11. Caldas JMP, Fonseca L, Almeida S, Almeida L. Escuela y diversidad sexual - ¿que realidad? Educ Rev (Belo Horizonte). 2012;28(3):14358. https://doi.org/10.1590/S0102-46982012000300007

12. Bortolini A. O sujeito homossexual como tema de aula: limites e oportunidades didáticas. Cad Pagu. 2015;45:479-501. https://doi.org/1 $0.1590 / 18094449201500450479$

13. Freitas DF, Coimbra S, Fontaine ANM. Resilience in LGB Youths: A Systematic Review of Protection Mechanisms. Paidéia (Ribeirão Preto). 2017;27(66):69-79. https://doi.org/10.1590/1982-43272766201709

14. Hadland SE, Yehia BR, Makadon HJ. Caring for Lesbian, Gay, Bisexual, Transgender, and Questioning Youth in Inclusive and Affirmative Environments. Pediatr Clin North Am. 2016;63(6):955-69. https://doi.org/10.1016/j.pcl.2016.07.001

15. Soliva TB, Silva Junior JB. Entre revelar e esconder: pais e filhos em face da descoberta da homossexualidade. Sex Salud Soc (Rio J.). 2014;17:124-48. https://doi.org/10.1590/1984-6487.sess.2014.17.08.a

16. Duncan DT, Hatzenbuehler ML. Lesbian, gay, bisexual, and transgender hate crimes and suicidality among a population-based sample of sexual-minority adolescents in Boston. Am J Public Health. 2014;104(2):272-8. https://doi.org/10.2105/AJPH.2013.301424

17. Sekoni AO, Gale NK, Manga-Atangana B, Bhadhuri A, Jolly K. The effects of educational curricula and training on LGBT-specific health issues for healthcare students and professionals: a mixed-method systematic review. J Int AIDS Soc. 2017;20(1):21624. https://doi. org/10.7448/IAS.20.1.21624

18. Nama N, MacPherson P, Sampson M, McMillan HJ. Medical students' perception of lesbian, gay, bisexual, and transgender (LGBT) discrimination in their learning environment and their self-reported comfort level for caring for LGBT patients: a survey study. Med Educ Online. 2017;22(1):1368850. https://doi.org/10.1080/10872981.2017.1368850

19. Kano M, Silva-Bañuelos AR, Sturm R, Willging CE. Stakeholders' Recommendations to Improve Patient-centered "LGBTQ" Primary Care in Rural and Multicultural Practices. J Am Board Fam Med. 2016;29(1):156-60. https://doi.org/10.3122/jabfm.2016.01.150205

20. Grosz AM, Gutierrez D, Lui AA, Chang JJ, Cole-Kelly K, Ng H. A Student-Led Introduction to Lesbian, Gay, Bisexual, and Transgender Health for First-Year Medical Students. Fam Med [Internet]. 2016 [acesso 2019 Jan 6];49(1):52-6. Disponível em: http://www.stfm.org/ FamilyMedicine/Vol49Issue1/Grosz52

21. Macapagal K, Bhatia R, Greene GJ. Differences in Healthcare Access, Use, and Experiences Within a Community Sample of Racially Diverse Lesbian, Gay, Bisexual, Transgender, and Questioning Emerging Adults. LGBT Health. 2016;3(6):434-42. https://doi.org/10.1089/ Igbt.2015.0124

22. Lee JH, Gamarel KE, Bryant KJ, Zaller ND, Operario D. Discrimination, Mental Health, and Substance Use Disorders Among Sexual Minority Populations. LGBT Health. 2016;3(4):258-65. https://doi.org/10.1089/lgbt.2015.0135

23. Sawning S, Steinbock S, Croley R, Combs R, Shaw A, Ganzel T. A first step in addressing medical education Curriculum gaps in lesbian-, gay-, bisexual-, and transgender-related content:The University of Louisville Lesbian, Gay, Bisexual, and Transgender Health Certificate Program. Educ Health (Abingdon). 2017;30(2):108-114. https://doi.org/10.4103/efh.EfH_78_16

24. Baig AA, Lopez FY, DeMeester RH, Jia JL, Peek ME, Vela MB. Addressing Barriers to Shared Decision Making Among Latino LGBTQ Patients and Healthcare Providers in Clinical Settings. LGBT Health. 2016;3(5):335-41. https://doi.org/10.1089/lgbt.2016.0014 